\title{
Adaptive Smoothing in fMRI Data Processing Neural Networks
}

\author{
Albert Vilamala \\ Kristoffer Hougaard Madsen \\ Lars Kai Hansen \\ Technical University of Denmark Danish Research Centre for Magnetic Resonance \\ alvmu@dtu.dk \\ kristofferm@drcmr.dk
Technical University of Denmark
lkai@dtu.dk

\begin{abstract}
Functional Magnetic Resonance Imaging (fMRI) relies on multi-step data processing pipelines to accurately determine brain activity; among them, the crucial step of spatial smoothing. These pipelines are commonly suboptimal, given the local optimisation strategy they use, treating each step in isolation. With the advent of new tools for deep learning, recent work has proposed to turn these pipelines into end-to-end learning networks. This change of paradigm offers new avenues to improvement as it allows for a global optimisation. The current work aims at benefitting from this paradigm shift by defining a smoothing step as a layer in these networks able to adaptively modulate the degree of smoothing required by each brain volume to better accomplish a given data analysis task. The viability is evaluated on real fMRI data where subjects did alternate between left and right finger tapping tasks.
\end{abstract}

\section{INTRODUCTION}

The use of non-invasive functional Magnetic Resonance Imaging (fMRI) techniques for determining brain activity requires a set of data processing steps that transforms raw data into validated elements suitable for statistical analysis.

Gaussian filter to average local voxel intensities is an important preprocessing step. Spatial smoothing serves several purposes [1]: local averaging reduces uncorrelated random noise in the voxel, hence increasing the Signal-to-Noise Ratio (SNR) leading to improved statistical power to detect true functional brain activation; also, spatial smoothing serves to eliminate unimportant anatomical details across subjects, that are preserved despite affine and non-linear transformations, which are common spatial normalisation steps in the pipeline; additionally, smoothing can ensure that assumptions typically made to enable multiple comparison correction using Random Field Theory (RFT) for locating brain activation are fulfilled.

Notice that the resolution of each brain volume is decreased by applying the smoothing step, meaning that an appropriate trade-off between the original volume and the degree of smoothing to be applied is sought. This compromise is governed by a single parameter stating the width of the Gaussian filter. In spite of the importance of this parameter, there is no established method to automatically select its most appropriate value for every situation, being often set according to best practices or relying on each scientist's expertise.

Previous attempts to build a system capable of adaptively smoothing brain volumes in fMRI include [2], which defines a Bayesian approach for the General Linear Model (GLM) able to determine the optimal amount of smoothing for each regressor; [3] and [4] which smooth the resulting statistical

978-1-5386-3159-1/17/\$31.00 @ 2017 IEEE parametric maps in a GLM instead of the input volumes, while providing an appropriate activation threshold based on the spatial correlation; and [5], that uses non-stationary spatial Gaussian Markov random fields, allowing the spatial extent of smoothing to vary across both space and time.

A recent work [6] proposes a change of paradigm by converting fMRI data processing pipelines into deep neural networks with the purpose of optimising the pipelines end-toend, instead of current state of the art, which optimises them locally at each step of the pipeline. In the same study, authors propose a generalisation of the Spatial Transformer Networks (STN) [7] as building blocks for these fMRI data processing neural networks.

In the current study, we use the aforementioned architecture to build a module able to adaptively select the most appropriate degree of smoothing for each volume in a data analysis task, allowing it to vary across time. This is a sharp contrast to previous work in the field, normally using preselected static smoothing or as a dynamic component within the GLM. In contrast to previous work [6], we here explicitly consider the main goals of spatial smoothing while designing and training the network. The provided solution can be used as a standalone module in regular fMRI pipelines or as part of fMRI data processing neural networks.

The rest of the document is structured as follows: Section $\Pi$ defines the architecture of the system and provides accurate implementation details, then we evaluate its performance for real fMRI brain data in Section [III, where subjects were instructed to do simple finger tapping tasks. Finally, we provide some concluding remarks and future research directions.

\section{MODEL}

Let $S=\left\{\mathbf{X}_{n}\right\}_{n=1}^{N}$ be a set containing fMRI brain volumes, each defined as $\mathbf{X} \in \mathbb{R}^{H \times W \times D}$ of height $H$, width $W$ and depth $D$. Our goal is to learn a function $\mathbf{Z}=s(\mathbf{X}, \sigma)$; $\mathbf{Z}$ being a volume of the same size as $\mathbf{X}$, corresponding to a spatially smoothed version of $\mathbf{X}$.

Following the architecture proposed in [6], $s(\mathbf{X}, \sigma)$ is learnt by using a neural network made up of two subnetworks: the transformation network, which smooths the input volumes by convolving them with a Gaussian filter, parameterised according to the values provided by the parameters network, that calculates the most adequate filter size for the current volume. Next we describe the system, depicted in Fig. 11, in more detail. 


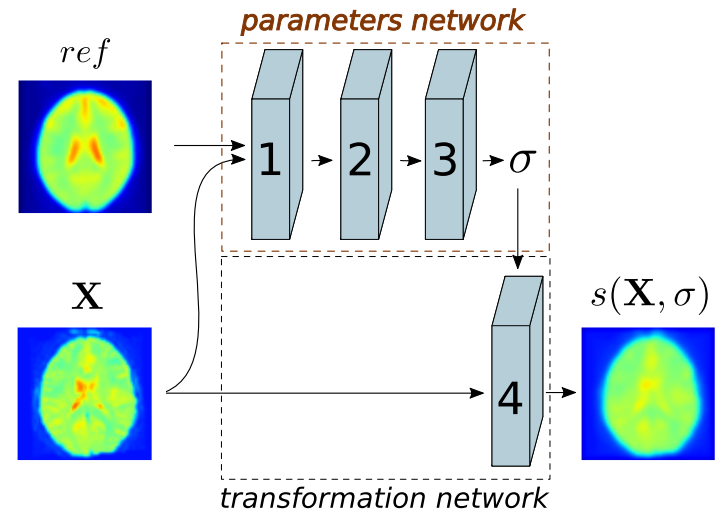

Fig. 1. Adaptive Smoothing neural network architecture.

\section{A. The transformation network}

Given an input volume and a value specifying the standard deviation parameter of the Gaussian filter $(\sigma)$, first the appropriate filter is constructed and then it is convolved by the input. These two steps are more precisely defined in the following.

1) Gaussian filter construction: Consider a smoothing function as a continuous isotropic 3-dimensional Gaussian function with standard deviation $\sigma$ :

$$
g(x, y, z ; \sigma)=\frac{1}{(\sqrt{2 \pi} \sigma)^{3}} \exp \left\{-\frac{x^{2}+y^{2}+z^{2}}{2 \sigma^{2}}\right\} .
$$

A filter $\mathbf{Q}$ of varying size is obtained by sampling from Eq. 1 at specific locations specified by a grid $G$, defined as $G_{i}=\left(x_{i}, y_{i}, z_{i}\right)$, where $-\lfloor(t \cdot \sigma+0.5) / 2\rfloor \leq x_{i}, y_{i}, z_{i} \leq$ $\lfloor(t \cdot \sigma+0.5) / 2\rfloor ; t$ being the number of standard deviations from the mean where the Gaussian function is truncated. Finally, all values in the filter are renormalised to sum 1.

Care must be taken for low $\sigma$ values. In particular, whenever $\sigma<1.5 / t$, the truncated discrete Gaussian function generates a single-cell $\mathbf{Q}$ filter, leading all partial derivatives of $\mathbf{Q}_{i, j, k}$ to be 0 ; hence, causing the backpropagation gradient to disappear. This misbehaviour is alleviated by stochastically adding 1.0 to $\sigma$ with probability $p$ (set to 0.1 in all our experiments) for values below the mentioned threshold. Renormalising the single-cell $\mathbf{Q}$ filter must be done with care, since dividing by the sum of all elements cancels $\sigma$ out, causing the gradient to disappear. An alternative solution is to clip the values of $\sigma$ provided by the parameters network above a certain threshold that avoids this problem altogether. Moreover, it is typically reasonable to apply a certain minimum smoothing, in particular when RFT assumptions are sought to be fulfilled, where a smoothing of minimally 2 times the voxel size is usually recommended [8].

2) Volume smoothing: For a given convolutional smoothing operator $\mathbf{Q} \in \mathbb{R}^{H^{\prime} \times W^{\prime} \times D^{\prime}}$, where $H^{\prime}<H, W^{\prime}<W$, $D^{\prime}<D$, spatially smoothing a volume can be accomplished by sliding the operator over it, performing a convolution in each location. Finally, each element of the resulting smoothed volume $\mathbf{Z} \in \mathbb{R}^{H \times W \times D}$ is computed as:

$$
\mathbf{Z}_{h, w, d}=\sum_{i=0}^{H^{\prime}} \sum_{j=0}^{W^{\prime}} \sum_{k=0}^{D^{\prime}} \mathbf{X}_{h+i, w+j, d+k} \cdot \mathbf{Q}_{i, j, k} .
$$

\section{B. The parameters network}

So far, we have been omitting the origin of the important $\sigma$ parameter in the Gaussian filter, which is calculated using the parameters network. This network is implemented as a conventional feed forward neural network, with a final regressor outputting a $\sigma$ value for each volume. More precisely, the first layer in the network is a centring layer, where a provided reference volume is subtracted from all brain volumes. The rationale for using an external reference has multiple explanations. First, the resulting network learns to account for relative deviations of each volume to the reference (being it the average brain of all subjects involved in the study or a Montreal Neurological Institute (MNI) template, as appropriate for the study). Secondly, given the high dimensionality of the data in this domain it is very unlikely that all volumes in a study can be loaded in memory and processed at a time: an external volume eases the task of keeping a global reference. The second layer is a $2 \times 2 \times 2$ maxpooling module, with the purpose of quantifying the average noise level in the volume. By definition, after centring the data, the average voxel intensity in each volume lays roughly around 0; a max-pooling layer only keeps the highest value in a neighbourhood, effectively increasing the average voxel intensity if noise is present in out-of-training sets. This has the effect of a multiplying factor to the value outputted by this network. The final layer is a fully-connected layer with a single output neuron with a softplus [9] activation function, ensuring non-negativity.

\section{Objective function}

In order to train the current module, we explicitly encode the goal of reducing inter-subject anatomical variability in the objective function. In particular, we seek to minimise this variability by smoothing individual volumes while penalising their deviation from the original volume. The final loss is computed as:

$$
L=\sum_{i}^{N}\left[s\left(\mathbf{X}_{i}, \sigma_{i}\right)-\frac{1}{N} \sum_{n}^{N} s\left(\mathbf{X}_{n}, \sigma_{n}\right)\right]^{2}+\lambda\left[\mathbf{X}_{i}-s\left(\mathbf{X}_{i}, \sigma_{i}\right)\right]^{2},
$$

$\lambda$ being a user-defined parameter controlling the trade-off between the smoothing level and deviation from original volumes (set to 0.5 in all our experiments).

\section{Data augmentation}

To overcome the limitation of small sample size that are usually available in this domain and reduce overfitting due to the number of parameters in the network, we strongly rely on data augmentation techniques to obtain realistic samples [10]. Specifically, given a volume from the training set $\mathbf{X}_{i}$, we generate a new sample volume $\mathbf{X}_{a}=\mathcal{T}_{\boldsymbol{\theta}}\left(\mathbf{X}_{i}\right)$; where $\mathcal{T}_{\boldsymbol{\theta}}$ is a spatial transformation function. In particular, we use a neural network composed of two 3D STN, the first one able to apply affine transformations to the input volumes and the second one using Thin Plate Splines (TPS) [11] to perform non-linear deformations (we use a sampling grid containing 192 free parameters as reference points). In contrast to their 
conventional use, the parameters of these modules are not calculated by their corresponding parameters networks, but they are randomly sampled from a multivariate Gaussian distribution $\boldsymbol{\theta} \sim \mathcal{N}(\boldsymbol{\mu}, \boldsymbol{\Sigma})$, where $\boldsymbol{\mu}$ and $\boldsymbol{\Sigma}$ are calculated by aligning the first volume of each pair of subjects in the training set. Formally, let $\mathbf{X}_{1}^{\alpha}$ and $\mathbf{X}_{1}^{\beta}$ be the first volume in the series for subjects $\alpha$ and $\beta$, respectively. Our goal is to optimise $\hat{\phi}_{\alpha \beta}=\underset{\phi}{\arg \min }\left[\mathbf{X}_{1}^{\alpha}-\mathcal{T}_{\phi}\left(\mathbf{X}_{1}^{\beta}\right)\right]^{2}$ for every pair of subjects, where $\mathcal{T}_{\phi}$ is the double 3D STN network defined in the previous paragraph. Then, $\boldsymbol{\mu}$ and $\boldsymbol{\Sigma}$ are set to the sample mean $\hat{\mu}$ and covariance $\hat{\Sigma}$ of matrix $\hat{\phi}$.

\section{EMPIRICAL EVALUATION}

\section{A. Materials}

We use fMRI data [12] from a simple sequential finger tapping paradigm, in which subjects alternated between 20 second blocks of left and right finger tapping separated by 10 seconds of rest. Data was recorded on a Siemens 3T scanner (Magnetom Trio) equipped with a standard birdcage headcoil. Each of the 29 subjects' data consisted of 240 volumes with $3 \mathrm{~mm}$ isotropic resolution sampled at a repetition time of 2.49 seconds. Further acquisition parameters can be found in [12]. After basic preprocessing steps including realignment and normalisation by standard settings in SPM12 (http://www. fil.ion.ucl.ac.uk/spm/software/spm12, revision 6685) each volume was labelled according to the left/right/rest condition starting from the second volume in each block.

\section{B. Artificially perturbed inputs}

1) Experimental setup: Out of the 29 subjects, the first 15 were used as the training set and the remaining 14 were set aside for testing. In this experiment, all 240 volumes per subject (normalised to $[0,1]$ using intra-subject extrema) were used irrespectively of their labelling. The fully-connected layer in the parameters network was initialised using Xavier initialisation [13]. Reference volume was constructed by averaging the first volume of all subjects in the training set. At each epoch, new data was created by randomly selecting 7 volumes from each subject in the training set and applying a random transformation to each one of them, as explained in Section II-D. The network was trained using Stochastic Gradient Descent (SGD) with Nesterov momentum for a number of internal epochs, after which new data was generated. All hyperparameters were selected using a Bayesian Optimisation (BO) strategy [14] validated on 7 subjects from the test set, randomly picking 15 volumes per subject and applying random transformations $\mathcal{T}_{\boldsymbol{\theta}}$. The whole procedure was repeated for the remaining 7 subjects in the test set. Optimal results for both rounds were obtained by hyperparameters: epochs $=50$, internal epochs $=75$, learning rate $=0.7$ and momentum $=0.9$.

2) Results: For assessing the capacity of our system to deal with noisy inputs, we randomly sample one volume from each of the 7 remaining subjects in the test set and add uniform white noise $\sigma_{n} \sim \mathcal{U}(-a, a)$ to each voxel, where $a=m \cdot \rho ; m=0.25$ being a predefined maximum noise level

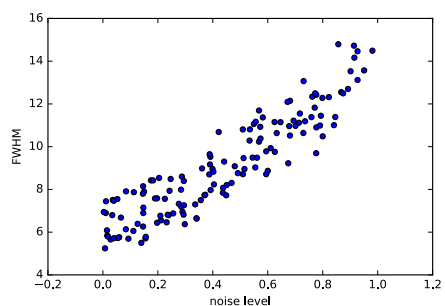

(a) White noise perturbation.

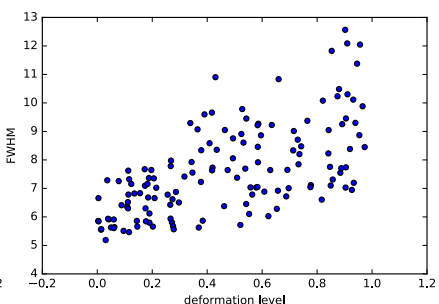

(b) Non-linear deformation.
Fig. 2. Correlation between the level of artificial perturbation applied to the volume $(\rho)$ and FWHM (in mm.) proposed by the network.

and $\rho \sim \mathcal{U}(0,1)$ expressing the deformation percentage to be applied to each new volume. Reference volume is created by averaging the first volume of each subject in the set. This procedure is being repeated 10 times, generating $2 \times 70$ new volumes with varying noise levels. These volumes are run through the network, which computes the corresponding filter size for each of the volumes. Results are converted to full width at half maximum (FWHM, a wide-spread measure of filter size). Fig. $2 \mathrm{a}$ shows the correlation between the noise level $\rho$ and the FWHM in mm.

Similarly, we use the previously introduced $\mathcal{T}_{\boldsymbol{\theta}}$ to generate 140 brain volumes affected by different deformation levels. The maximum parameter levels are set to 0.05 for translations, shearing and scaling, $\pi / 32$ for rotations, and 0.05 for nonlinear warpings. Fig. $2 \mathrm{~b}$ depicts the correlation between the different deformation levels $\rho$ and the resulting FWHM in mm.

\section{Inter-subject anatomical differences}

In this experiment we want to evaluate the capacity of our pre-trained system to deal with the anatomical differences between subjects in the test set. We run our pre-trained network over all volumes in the test set, using the average of subjects' first volume as a reference. Table $[$ shows the average sum of squares difference between each smoothed subject's brain volume and the reference volume smoothed accordingly (i.e. first term of Eq. 2). The penalty term is also calculated with the purpose to quantify the degree of loss incurred by each subject due to smoothing. Results show that when no smoothing is used (raw column) the anatomical differences are highest, while when standard-practise $8 \mathrm{~mm}$. smoothing is applied, differences are diminished at the price of higher penalty to the raw image; adaptive smoothing presents a better trade-off between the two. Anat. column in FWHM shows the average smoothing the network calculates for each subject.

Looking at Fig. 3, the system shows general consistency in assigning varying smoothing levels to volumes according to their distance to the reference volume. Likewise, it is interesting to notice that although the use of different smoothing accounting for inter-subject dissimilarities seems plausible, intra-subject differences are quite minimal, therefore, there is no real need to use a per-volume smoothing at this stage.

\section{Brain decoding task}

The strongest point of the proposed adaptive smoothing is its capacity to adjust the degree of smoothing to each specific 
TABLE I

INTER-SUBJECT ANATOMICAL DIFFERENCES AND ASSIGNED SMOOTHING.

\begin{tabular}{c|rrr|rr|rrr|rr} 
& \multicolumn{3}{|c|}{ Differences } & \multicolumn{2}{|c|}{ Penalty } & \multicolumn{3}{|c}{ FWHM } & \multicolumn{2}{|c}{ Acc. (in \%) } \\
\# subj. & raw & $8 \mathrm{~mm}$ & adap. & $8 \mathrm{~mm}$ & adap. & anat. & dec. & dec_n & $8 \mathrm{~mm}$ & adap. \\
16 & 16.1 & 9.3 & 10.8 & 18.0 & 12.8 & 5.8 & 3.7 & 9.8 & 58 & 58 \\
17 & 21.5 & 16.5 & 16.6 & 17.1 & 16.4 & 7.5 & 4.1 & 10.8 & 61 & 66 \\
18 & 15.3 & 7.7 & 9.5 & 17.8 & 12.6 & 5.7 & 3.3 & 9.7 & 62 & 56 \\
19 & 17.8 & 9.7 & 11.7 & 18.0 & 12.8 & 5.6 & 3.7 & 9.7 & 65 & 62 \\
20 & 19.5 & 11.5 & 12.1 & 17.7 & 16.0 & 6.8 & 4.8 & 10.6 & 61 & 65 \\
21 & 16.7 & 11.2 & 12.3 & 16.4 & 12.3 & 6.0 & 5.0 & 10.5 & 58 & 63 \\
22 & 17.1 & 9.7 & 9.9 & 18.0 & 17.2 & 7.4 & 3.7 & 11.2 & 63 & 66 \\
23 & 19.1 & 13.2 & 13.4 & 16.8 & 16.1 & 7.4 & 3.7 & 11.2 & 55 & 56 \\
24 & 20.5 & 16.4 & 17.3 & 15.9 & 10.9 & 5.3 & 5.1 & 10.1 & 56 & 62 \\
25 & 16.1 & 9.6 & 10.1 & 17.8 & 15.8 & 6.7 & 3.1 & 11.1 & 53 & 62 \\
26 & 17.0 & 11.2 & 12.4 & 17.1 & 12.4 & 5.8 & 4.5 & 10.4 & 59 & 65 \\
27 & 19.7 & 13.3 & 13.8 & 18.3 & 16.3 & 6.7 & 2.6 & 10.7 & 59 & 64 \\
28 & 18.2 & 10.1 & 10.6 & 17.6 & 16.3 & 7.1 & 3.5 & 11.3 & 62 & 62 \\
29 & 15.4 & 8.7 & 10.4 & 16.5 & 11.5 & 5.6 & 3.3 & 10.3 & 61 & 60 \\
Avg. & 17.9 & 11.3 & 12.2 & 17.4 & 14.2 & 6.4 & 3.9 & 10.5 & 59 & 62
\end{tabular}

Differences show the average sum of squares distance between each subject and reference volume for a variety of smoothings: raw data, fixed $8 \mathrm{~mm}$. and using our adaptive method. Similarly, Penalty expresses the same measure between smoothed and raw instances. FWHM shows the kernel width selected by our adaptive method for different analytical tasks: inter-subject anatomical differences, brain decoding and decoding with noise. Acc. column reports the obtained accuracies for the noisy brain decoding task.

data analysis task, as part of a multilayer neural network. In this section, we add a decoding module after the smoothing one aiming at classifying each brain volume as the state where the subject was finger tapping her right or left hand; hence, brain volumes labelled as rest were discarded, using only 200 volumes per subject. The decoding module is composed of a fully-connected layer with single linear output, to which a variant of batch-normalisation [15] is applied before a sigmoid activation. Importantly, all 200 volumes of the same subject conform the mini-batch, which coupled to the offline nature of the experiments, allows for using current batch statistics, being it training, validation or testing. All settings and training procedures were defined as in the previous experiment (i.e., 15 subjects for training, 7 for validation and 7 for test), but using the pre-trained weights as a starting point for the parameters network. No data augmentation was used and binary crossentropy between predicted values and targets was the loss function of choice. Network was trained, allowing fine-tune of the parameters network, using SGD with hyperparameters set to be epochs $=100$, learning rate $=0.01$.

Here, we allow the adaptive smoothing to freely choose the most adequate smoothing (i.e., adapting weights in the parameters network) providing the highest classification accuracy. Notice that we are not aiming at improving accuracy in this setting (due to the powerful motor activation paradigm, the classification can be obtained almost perfectly without smoothing), but we rather want to observe the behaviour of the adaptive smoothing module. As expected, dec. column in Table I shows the average smoothing parameter for each subject, which tends to vanish for the current task. In order to challenge the network, Gaussian white noise with $\sigma=0.25$ is artificially added to the input volumes, forcing the smoothing kernel to be widened: $d e c \_n$ column shows the most appropriate average smoothing for each subject, and last two columns present the corresponding classification accuracies. Although there seems to be a slight improvement in classification accuracy when our method is employed, further experiments should be done

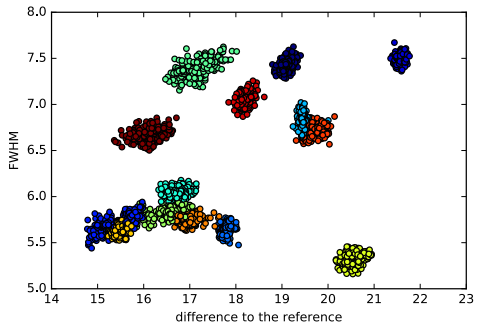

Fig. 3. Correlation between volume difference to the reference and FWHM (in $\mathrm{mm}$.) proposed by the network. Each colour represents a different subject.

in datasets encoding tasks highly dependent on the smoothing step to appreciate the extent of our method.

\section{CONCLUSion}

Using a novel implemented spatial smoothing module, we here demonstrate that it is possible to construct differentiable modules of neural networks that form flexible and dynamic analysis pipelines for fMRI data. As a proof of concept we additionally show that it is feasible to train such networks end-to-end, which is an important step towards truly optimal processing pipelines. Future work will focus on the development of a standard fMRI pipeline in form of a neural network, including determining spatial location activations.

\section{ACKNOWLEDGMENT}

This project has received funding from the European Union's Horizon 2020 research and innovation programme under the Marie Sklodowska-Curie grant agreement No 659860. We gratefully acknowledge the support of NVIDIA Corporation with the donation of the GPUs used for this research.

\section{REFERENCES}

[1] M. A. Lindquist, "The statistical analysis of fMRI data," Statistical Science, vol. 23, no. 4, pp. 439-464, 112008.

[2] W. D. Penny et al., "Bayesian fMRI time series analysis with spatial priors," NeuroImage, vol. 24, no. 2, pp. 350-362, 2005.

[3] K. Tabelow et al., "Analyzing fMRI experiments with structural adaptive smoothing procedures," Neurolmage, vol. 33, no. 1, pp. 55-62, 2006.

[4] I. Almodóvar-Rivera and R. Maitra, "FAST adaptive smoothing and thresholding for improved activation detection in low-signal fMRI," stat, vol. abs/1702.00111, 2017.

[5] Y. R. Yue et al., "Adaptive spatial smoothing of fMRI images," Statistics and Its Interface, vol. 3, no. 1, pp. 3-13, 2010.

[6] A. Vilamala, K. H. Madsen, and L. K. Hansen, "Towards end-toend optimisation of functional image analysis pipelines," CoRR, vol. abs/1610.04079, 2016.

[7] M. Jaderberg et al., "Spatial transformer networks," CoRR, vol. abs/1506.02025, 2015.

[8] K. Worsley and K. Friston, "Analysis of fMRI time-series revisitedagain," NeuroImage, vol. 2, no. 3, pp. 173-181, 1995.

[9] C. Dugas et al., "Incorporating second-order functional knowledge for better option pricing," in NIPS, 2001, pp. 451-457.

[10] S. Hauberg et al., "Dreaming more data: Class-dependent distributions over diffeomorphisms for learned data augmentation," in AISTATS, 2016.

[11] F. L. Bookstein, "Principal warps: Thin-plate splines and the decomposition of deformations," IEEE Trans. Pattern Anal. Mach. Intell., vol. 11, no. 6, pp. 567-585, Jun. 1989.

[12] P. M. Rasmussen et al., "Visualization of nonlinear kernel models in neuroimaging by sensitivity maps," NeuroImage, vol. 55, no. 3, pp. 1120-1131, 2011.

[13] X. Glorot and Y. Bengio, "Understanding the difficulty of training deep feedforward neural networks," in AISTATS, 2010.

[14] J. Snoek, H. Larochelle, and R. P. Adams, "Practical bayesian optimization of machine learning algorithms," in NIPS, 2012, pp. 2951-2959.

[15] S. Ioffe and C. Szegedy, "Batch normalization: Accelerating deep network training by reducing internal covariate shift," CoRR, vol. abs/1502.03167, 2015. 